\title{
Primary mediastinal germ cell tumours: real world experience in the low middle income (LMIC) setting
} \author{
Josh Thomas Georgy ${ }^{1}$, Raju Titus Chacko ${ }^{1}$, Aparna Irodi ${ }^{4}$, Bijesh Yadav ${ }^{5}$, Subhashini John ${ }^{6}$ and Ashish Singh ${ }^{1}$ \\ ${ }^{1}$ Department of Medical Oncology, Christian Medical College and Hospital Vellore, Vellore 632004, India \\ ${ }^{2}$ Department of Thoracic Surgery, Christian Medical College and Hospital Vellore, Vellore 632004, India \\ ${ }^{3}$ Department of Pathology, Christian Medical College and Hospital Vellore, Vellore 632004, India \\ ${ }^{4}$ Department of Radiology, Christian Medical College and Hospital Vellore, Vellore 632004, India \\ ${ }^{5}$ Department of Biostatistics, Christian Medical College and Hospital Vellore, Vellore 632004, India \\ ${ }^{6}$ Department of Radiotherapy, Christian Medical College and Hospital Vellore, Vellore 632004, India
}

Anjana Joel ${ }^{1}$, Namrata Mathew ${ }^{1}$, Shalom Sylvester Andugala², Sherin Daniel ${ }^{3}$, Birla Roy Gnanamuthu², Ajoy Oommen John ${ }^{1}$,

\section{Abstract}

Purpose: Primary mediastinal germ cell tumours (PMGCTs) are rare; with limited data available about their outcomes and optimal treatment in the low middle income countries setting. We studied the clinical profile of patients with PMGCT treated at our centre in order to estimate their survival outcomes and to identify prognostic factors affecting the same.

Patients and methods: Fifty-seven patients with PMGCTs treated between April 2001 and June 2019 were included. Baseline characteristics, details of first line chemotherapy, response rates, toxicity and surgical outcomes were noted. Progression-free survival (PFS) and overall survival (OS) were estimated using the Kaplan-Meier method.

Results: Among 57 male patients (seminoma $=20$ and nonseminomatous $=37$ ), the median follow-up was 10 months (range: 1-120 months). For mediastinal seminoma, 9 (45\%) and 11 (55\%) patients had good and intermediate risk disease, respectively. Nineteen patients (95\%) received BEP (Bleomycin, etoposide and cisplatin) chemotherapy. $94.7 \%$ had partial responses and median event-free survival was not reached. All patients were alive and disease free at 2 years. For primary mediastinal nonseminomatous germ cell tumours (PMNSGCTs), all patients were poor risk. Thirty-four (91.8\%) received BEP/ EP chemotherapy as first line. Responses were PRM+ (partial response with elevated markers) in 7 (20.5\%) and PRM- in 12 (35.2\%). The incidence of febrile neutropenia was $50 \%$ and $55.8 \%$ in seminole and PMNSGCT, respectively. The median OS was 9.06 months and median PFS was 4.63 months for PMNSGCT. The proportion of patients alive at 1 year and 2 years were $35 \%$ and $24.3 \%$, respectively.

Conclusion: Primary mediastinal seminomas are rarer and have better survival outcomes. Treatment of PMNSGCT is still a challenge and is associated with poorer survival outcomes.

Keywords: Primary mediastinal germ cell tumour, mediastinal seminoma, primary mediastinal nonseminomatous germ cell tumour, low middle income countries

Correspondence to: Dr Ashish Singh Email: todrashish@gmail.com

ecancer 2021, 15:1186

https://doi.org/10.3332/ecancer.2021.1186

Published: $11 / 02 / 2021$

Received: 13/11/2020

Publication costs for this article were supported by ecancer (UK Charity number 1176307).

Copyright: (c) the authors; licensee ecancermedicalscience. This is an Open Access article distributed under the terms of the Creative Commons Attribution License (http:// creativecommons.org/licenses/by/3.0), which permits unrestricted use, distribution, and reproduction in any medium, provided the original work is properly cited. 


\section{Background}

Primary mediastinal germ cell tumours (PMGCTs) account for $<5 \%$ of all GCTs [1]. They are classified as 'intermediate or poor risk' as per the International Germ Cell Consensus Collaborative Group (IGCCCG) just based on their origin in the mediastinum [2]. Though mediastinal seminomas have excellent cure rates and survival outcomes, primary mediastinal nonseminomatous germ cell tumours (PMNSGCTs) which are histologically and cytogenetically similar to gonadal NSGCTs have a dismal prognosis. This is attributed to their more extensive stage at diagnosis, a poorly understood disease biology, compounded by a lack of targetable mutations and a lack of response to immunotherapy as demonstrated in refractory testicular GCTs [3-5].

Mediastinal seminoma responds to initial chemotherapy (BEP/VIP) and relapses though infrequent are often salvaged with second line chemotherapy. The primary treatment of PMNSGCT is multimodality; traditionally induction chemotherapy (BEP/VIP) followed by surgical excision, after documentation of both a radiological and serological response [5]. In the recurrent/refractory setting, salvage chemotherapy with or without high dose therapy (HDT) is standard of care [5]. In low middle income countries (LMIC) like ours, the management of GCTs is more challenging due to the higher proportion of patients with extra mediastinal and extra thoracic involvement at diagnosis of PMGCTs, higher rates of treatment abandonment and attrition, limited access to surgical expertise and salvage chemotherapy with HDT/autologous stem cell transplantation (ASCT) at relapse [6-9]. We reviewed our institutional experience with PMGCTs with an intent to determine the differences in presentation, treatment course and survival outcomes of these patients in the real world LMIC setting.

\section{Materials and methods}

\section{Study population}

We examined the medical records of consecutive adult patients (age >16 years) with PMGCTs treated at our centre from April 2001 to June 2019.

\section{Baseline characteristics}

We collected the following information: demographics, method of preoperative diagnosis/biopsy report, alpha fetoprotein (AFP), betahuman chorionic gonadotrophin (Beta-HCG) and lactate dehydrogenase (LDH) tumour marker values at diagnosis and radiological information. Image guided biopsy was done for all patients with normal serum AFP ( $<5.5 \mathrm{IU} / \mathrm{mL}$ ), and beta-HCG $<200 \mathrm{~m}$-IU/mL, who were suspected to have primary mediastinal seminoma. It was not mandatory for patients with an anterior mediastinal mass with elevated serum AFP or beta-HCG > $200 \mathrm{~m}$-IU/mL, diagnosed to have PMNSCGT, to undergo an image guided biopsy of the mediastinal mass prior to initiation of chemotherapy. All patients underwent contrast enhanced CT imaging of the thorax +/- abdomen/pelvis, prior to and after completion of all chemotherapy. End of treatment fluorodeoxy glucose-positron emission tomography (FDG-PET) CT (EOT PET CT) was done after completion of chemotherapy only in patients with seminoma. All patients were stratified as good, intermediate or poor risk as per the IGCCCG classification based on their serum markers and tumour type. The last date of outpatient clinic visit was taken as the date of last follow up. For patients not returning to hospital, the follow up was by telephonic interview.

\section{Treatment details}

The details of initial chemotherapy regimens, duration of treatment and best response (pre- and post-chemotherapy tumour markers and imaging) were collected. Radiological response assessment was performed using radiological assessment according to the Response Evaluation Criteria in Solid Tumours version 1.1. Overall disease response was documented for patients with PMNSGCT based on the combination of radiological response and tumour markers as: complete response (CR), partial response with normal tumour markers (PRM-), partial response with plateau of tumour markers (PRM+) and progressive disease (PD). Adverse events during treatment were documented and graded using the Common Terminology Criteria for Adverse Events, version 4.02. 
The surgical details, tumour stage, surgical pathology (including preoperative biopsy and postresection histology) of patients with PMNSCGT who underwent post chemotherapy surgical resection were collected. Post-operative chemotherapy was administered to patients whose surgical histopathology showed residual viable tumour. Among patients who relapsed or were refractory to first line chemotherapy, the details of salvage chemotherapy and outcomes were collected.

\section{Survival outcomes}

We defined an event as disease relapse or progression or death due to any cause; if the death occurred before disease progression; or the last follow-up date, whichever was earlier. Patients who were lost to follow-up were censored on the date of their last follow-up. Data was censored on 30 April 2020. We calculated event-free survival (EFS) from the date of initiation of treatment to the date of the event and overall survival (OS) was calculated from the date of histopathological diagnosis to the date of death. Patients who were still alive at the end of the study were censored on the date of the last follow up. We included patients who abandoned treatment in our survival analysis; since their inclusion is more reflective of the real world scenario [10].

\section{Statistical analysis}

Data was entered and analysed using SPSS Version 23. Categorical and continuous variables were summarised using descriptive statistics. Disease-free survival and OS were calculated from the time of diagnosis using the Kaplan-Meir method. Time-to-event analysis was done using the Kaplan-Meier estimator, and hazard ratios were calculated using the Cox proportional model.

\section{Results}

Fifty-seven patients were diagnosed with mediastinal GCT at our centre from April 2001 to June 2019. Among these, 20 were diagnosed with primary mediastinal seminoma and 37 as PMNSGCT based on elevated tumour markers and pre-operative biopsy (Figure 1).

\section{Patient characteristics}

All the patients were male. The median age at diagnosis was 25 years (13-44) and 22 years (15-49) for seminoma and PMNSGCT, respectively. The commonest presenting symptoms were cough, breathlessness and systemic symptoms in both groups of patients (Table 1). There were distant metastases in $10 \%$ and $40 \%$ of patients with mediastinal seminoma and PMNSGCT, respectively. None of our patients had associated leukaemia or myelodysplastic syndrome or Klinefelter's syndrome, indicating the rarity of these associations (Tables 1 and 2 ).

\section{Histologic and tumour marker evaluations}

Among patients with PMNSGCT, the median serum AFP, B-HCG, LDH at diagnosis were 13,682 IU/mL (range: 0.69-89,500), 60.3 m-IU/mL (range: 0.8-81,940) and $912 \mathrm{U} / \mathrm{L}$ (range: 265 to 4,341), respectively. Preoperative biopsy was done in all patients with mediastinal seminoma. The baseline B-HCG was >1,000 m-IU/mL in 6 (16.2\%) patients; all with PMNSGCT (Table 1).

\section{IGCCCG risk stratification}

Among patients with mediastinal seminoma, 11 (55\%) belonged to 'intermediate risk' and 9 (45\%) to 'good risk' as per IGCCCG risk criteria. All 37 patients with PMNSGCT were stratified as 'poor risk' based on IGCCCG criteria (Table 3). 


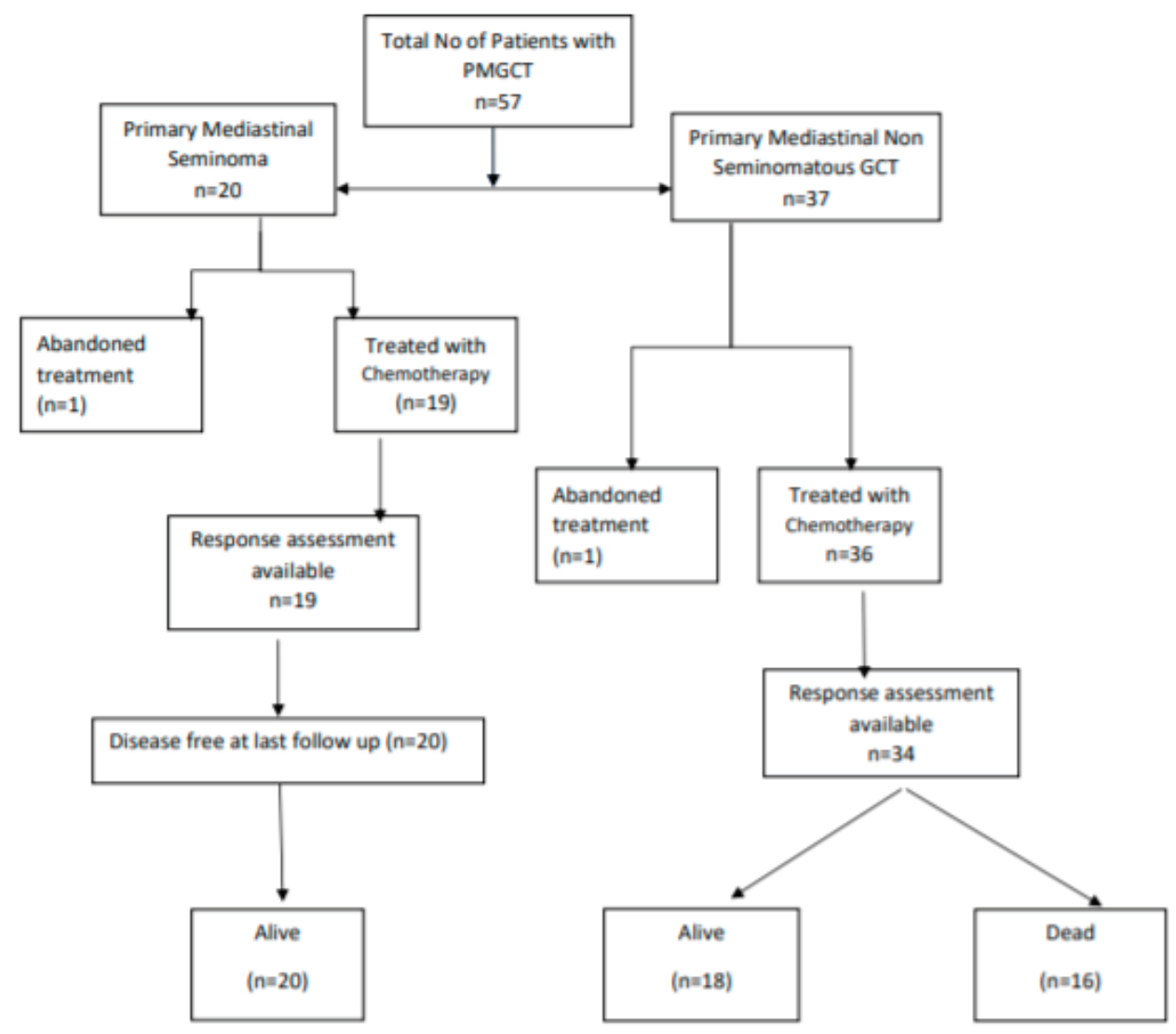

Figure 1. STROBE diagram for study population (STROBE, strengthening the reporting of observational studies in epidemiology).

\section{Chemotherapy details}

All patients received cisplatin-based chemotherapy as first line treatment. Nineteen patients with mediastinal seminoma received either three cycles of BEP or four cycles of EP chemotherapy. Among patients with PMNSGCT, majority (91.9\%) received either four cycles of BEP or three cycles BEP followed by one cycle EP and two patients received non bleomycin containing first line chemotherapy (VIP/TIP (Paclitaxel, ifosfamide and cisplatin)). Prophylactic pegfilgrastim on Day 6 was used in patients receiving EP chemotherapy. Among patients on BEP chemotherapy, routine pegfilgrastim was not used and filgrastim was used as indicated during episodes of febrile neutropenia or during delayed recovery of white blood cell counts. One patient with seminoma and two with PMNSGCT received one cycle of pre-phase chemotherapy with single agent carboplatin AUC 5 in view of poor Eastern Cooperative Oncology Group (ECOG) performance status; before initiation of standard chemotherapy. The median number of cycles of first line chemotherapy was four in both groups (Table 4).

\section{Post chemotherapy evaluation and responses}

Majority of patients (75\%) with seminoma had a partial response as per radiological assessment. Ten patients with seminoma underwent EOT PET CT; all of whom were interpreted as PET positive. These patients, however, have remained on surveillance with subsequent CT imaging showing further decrease in size of the residual mass and are all currently in remission.Among PMNSGCT, response assessment was 
based on both radiology and tumour markers. There was one patient with a radiological CR with normal tumour markers. Partial radiological response with normal tumour markers (PRM-) and with plateau of tumour marker decline (PRM+) was seen in 12 (35.2\%) and 7 (20.5\%) patients, respectively. Eleven (29.7\%) had primary refractory disease. Among the patients with PRM+ $(n=7)$, three had tumours which were more necrotic and cystic on CT imaging. Among 12 patients with PRM-, six had tumours which were more necrotic and cystic on CT imaging.

\section{Post chemotherapy management}

Fourteen patients with seminoma remained on surveillance. Two patients were given radiotherapy to the residual mediastinal mass and four patients underwent surgery; all their surgical biopsies showed extensive fibrosis with necrosis. Following the introduction of the SEMPET trial, EOT-PET CT was done in 10 patients; at a median time of 10 weeks (range: 8-16 weeks) from the date of last chemotherapy. All these patients were interpreted as EOT PET positive based on the standardised uptake value uptake (as per the SEMPET definition) but remained on surveillance; subsequent imaging has shown further decrease in size of the residual mediastinal mass. Post chemotherapy surgery was done in 12 (32.4\%) patients with PMNSGCT (Table 5). Surgical resection was R0, R1 and R2 in 33\%, 16\% and 25\%, respectively. There was viable tumour in two patients (16\%) and in three patients the surgery was done at another centre and the histopathology was not available. The remaining seven patients had necrosis or mature teratoma. Six patients (17.6\%) did not undergo surgery despite being in remission. Two patients had minimal low volume residual disease and chose to remain on follow up, two had residual multiple cystic liver lesions and bilateral lung metastases, respectively, and two refused surgery.

Table 1. Baseline characteristics $(N=57)$.

\begin{tabular}{|c|c|c|}
\hline & Seminoma $(n=20)$ & Nonseminoma $(n=37)$ \\
\hline Median age in years (range) & $25(13-44)$ & $22(15-49)$ \\
\hline Gender, Male & $20(100 \%)$ & $37(100 \%)$ \\
\hline Patients who underwent pretreatment biopsy & 20 (100\%) & 31 (83.8\%) \\
\hline Yolk sac histology & NA & $11(29.7 \%)$ \\
\hline Smokers & $4(20 \%)$ & $5(13.5 \%)$ \\
\hline Other risk factors & Crypto-orchidism seen in two individuals & Autism seen in one individual \\
\hline $\begin{array}{l}\text { Presenting symptoms (\%) } \\
\text { - Cough } \\
\text { - Breathlessness } \\
\text { - Systemic symptoms (fever/LOW/LOA) } \\
\text { - Chest pain } \\
\text { - Voice change } \\
\text { - Others }\end{array}$ & $\begin{array}{l}11(55 \%) \\
10(50 \%) \\
5(25 \%) \\
11(55 \%) \\
2(10 \%) \\
7(35 \%)\end{array}$ & $\begin{array}{c}20(54.1 \%) \\
12(34.1 \%) \\
13(35.1 \%) \\
18(45.6 \%) \\
2(5.4 \%) \\
9(24.3 \%)\end{array}$ \\
\hline \multicolumn{3}{|l|}{ ECOG at diagnosis (\%) } \\
\hline - 1 & $18(90 \%)$ & $21(56.8 \%)$ \\
\hline - 2 & $1(5 \%)$ & $10(27 \%)$ \\
\hline - 3 & $1(5 \%)$ & $3(8.1 \%)$ \\
\hline - 4 & 0 & $3(8.1 \%)$ \\
\hline Median haemoglobin (gm\%) (range) & $13.05(10.67-16.5)$ & $11.4(7.6-16.1)$ \\
\hline Median AFP (median, range) & $1.67(0.8-9.59)$ & $13,682(0.69-89,500)$ \\
\hline Median HCG (median, range) & $32.1(0.2-466)$ & $60.3(0.8-81,940)$ \\
\hline Median LDH (median, range) & $1,366(288-4,733)$ & $912(265-4,341)$ \\
\hline
\end{tabular}

LOW: loss of weight; LOA: loss of appetite 
Table 2. Disease extent $(n=57)$.

\begin{tabular}{|c|c|c|}
\hline & Seminoma $(n=20)$ & Nonseminoma $(n=37)$ \\
\hline Median tumour size (in cm) (range) & $\begin{array}{c}\text { Median } 12.5 \\
(7-23)\end{array}$ & $\begin{array}{l}\text { Median } 12 \\
(5-20)\end{array}$ \\
\hline \multicolumn{3}{|l|}{ Nodes involved (\%) } \\
\hline - Mediastinal & $2(10)$ & $7(18.9)$ \\
\hline - Nonmediastinal & $7(35)$ & $5(13.5)$ \\
\hline - None & $11(55)$ & $25(67.6 \%)$ \\
\hline SVCO present (\%) & $8(40)$ & 9 (24.3\%) \\
\hline \multicolumn{3}{|l|}{ Other local complications (\%) } \\
\hline - Pericardial & $6(30)$ & $11(29.7 \%)$ \\
\hline - Pleural or lung & $5(25)$ & $17(45.9 \%)$ \\
\hline - Vocal cord palsies & $2(10)$ & $1(2.7 \%)$ \\
\hline - Others (atrial, rib, spinal cord) & $1(5)$ & 9 (24.3\%) \\
\hline - None & $9(45)$ & 7 (18.9) \\
\hline - Two or more of the above & $3(15)$ & $6(16 \%)$ \\
\hline Distant metastases present, outside mediastinum (\%) & $2(10 \%)$ & $15(40 \%)$ \\
\hline \multicolumn{3}{|l|}{$\begin{array}{l}\text { Sites of distant metastases } \\
\text { (osseous, visceral, both, none) }\end{array}$} \\
\hline - Osseous & 1 & $2(5.4 \%)$ \\
\hline - Visceral ${ }^{a}$ & 1 & $8(21.6 \%)$ \\
\hline - None & 0 & $5(13.5 \%)$ \\
\hline - Both osseous and visceral & 0 & $22(59.5 \%)$ \\
\hline Brain metastases present & 0 & $3(8.1 \%)$ \\
\hline $\mathrm{HCG} \geq 1,000$ & $x$ & $5(13.5 \%)$ \\
\hline \multicolumn{3}{|l|}{ IGCCCG risk staging (\%) } \\
\hline - Good risk & $9(45 \%)$ & 0 \\
\hline - Intermediate risk & $11(55 \%)$ & 0 \\
\hline - Poor risk & $x$ & $37(100 \%)$ \\
\hline
\end{tabular}

SVCO: superior vena cava obstruction

${ }^{\mathrm{a}}$ One patient had a $3 \mathrm{~mm}$ indeterminate lung nodule.

\section{Toxicity}

Febrile neutropenia rates were similar across both seminoma and PMNSGCT groups. The incidence of all grades of anaemia and thrombocytopenia, including Grade 3 and 4 was higher in the PMNSGCT subset (Table 3). The incidence of Grade 3/Grade 4 neutropenia was $45 \%$ ( 9 out of 20 patients) and 54\% (20 out of 37 patients) in seminoma and PMNSGCT, respectively. There were no deaths attributed to febrile neutropenia in either group. The incidence of Grade 3 acute kidney injury was 38.8\% and 20.5\% among seminoma and PMNSGCT subgroups, respectively. None of these patients required haemodialysis. 
Table 3. First line chemotherapy, responses and toxicity.

\begin{tabular}{|c|c|c|}
\hline & Seminoma $(n=20)$ & Nonseminoma $(n=37)$ \\
\hline Prephase & $1(5 \%)$ & $2(5.4 \%)$ \\
\hline Median number of cycles of first line chemotherapy & $3.95(0$ to 6$)$ & 4 (1to 6) \\
\hline Abandoned treatment & $1(5 \%)$ & $1(2.7 \%)$ \\
\hline $\begin{array}{l}\text { First line chemotherapy regimen } \\
\text { - EP } \\
\text { - VIP } \\
\text { - BEP }\end{array}$ & $\begin{array}{c}6 \\
0 \\
13\end{array}$ & $\begin{array}{c}13 \\
2 \\
21\end{array}$ \\
\hline $\begin{array}{l}\text { Tumour marker trend at completion of first line } \\
\text { chemotherapy }\end{array}$ & $N=19$ & $N=34$ \\
\hline - Normal & $16(84.2 \%)$ & $10(27 \%)$ \\
\hline - Plateau & 0 & $19(51.3 \%)$ \\
\hline - High & $2(10.5 \%)^{a}$ & $2(5.8 \%)$ \\
\hline - Not done & $1(8.3 \%)$ & $3(8.1 \%)$ \\
\hline Radiological response after first line chemotherapy & $N=19$ & $N=34$ \\
\hline - $\mathrm{CR}$ & $1(5.3 \%)$ & $1(2.7 \%)$ \\
\hline - $\mathrm{PR}$ & $18(94.7 \%)$ & $17(45.9)$ \\
\hline - SD & 0 & $2(5.4 \%)$ \\
\hline - $\mathrm{PD}$ & 0 & $11(29.7 \%)$ \\
\hline - Not done & 0 & $6(16.2 \%)$ \\
\hline $\begin{array}{l}\text { Overall disease response (imaging + markers) after } \\
\text { first line chemotherapy }\end{array}$ & $N=19$ & $N=34$ \\
\hline - $\mathrm{CR}$ & $1(5.3 \%)$ & $1(2.9 \%)$ \\
\hline - PRM- & $16(84.2 \%)$ & $12(35.2 \%)$ \\
\hline - $\mathrm{PRM+}$ & $2(10.5 \%)$ & $7(20.5 \%)$ \\
\hline - $\mathrm{PD}$ & 0 & $10(29.4 \%)$ \\
\hline - Not done ${ }^{b}$ & 0 & $4(12 \%)$ \\
\hline \multicolumn{3}{|l|}{ Haematologic toxicities } \\
\hline - Febrile neutropenia & $9(50 \%)$ & $19(55.8 \%)$ \\
\hline - Neutropenia (G3 or G4) & $9(45 \%)$ & $20(54 \%)$ \\
\hline - Anaemia (any grade) & $8(44.4 \%)$ & $26(76.4 \%)$ \\
\hline - Anaemia (G3 or G4) & $2(11.1 \%)$ & $22(64.7 \%)$ \\
\hline - Thrombocytopenia (any grade) & $9(50 \%)$ & $20(58.8 \%)$ \\
\hline - Thrombocytopenia (any grade/G3 or G4) & $4(22.2 \%)$ & $16(47 \%)$ \\
\hline \multicolumn{3}{|l|}{ Non haematologic toxicities } \\
\hline - Pneumonitis & $1(5.5 \%)$ & $2(5.8 \%)$ \\
\hline - Peripheral neuropathy & 0 & $3(8.8 \%)$ \\
\hline - Acute kidney injury/renal failure Grade 3 & $7(38.8 \%)$ & $7(20.5 \%)$ \\
\hline
\end{tabular}

a Persistent elevated LDH in seminoma.

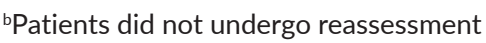


Table 4. Post first line chemotherapy course in mediastinal seminoma.

\begin{tabular}{|l|c|}
\hline & Seminoma \\
\hline Surveillance & $14(90 \%)$ \\
\hline Radiation therapy & $2(10 \%)$ \\
\hline Surgical resection & 4 \\
\hline EOT PET done & 10 \\
\hline Median time from last chemo to PET CT & 12 weeks (8-16 weeks) \\
\hline EOT PET CT interpretation as 'positive' & 10 \\
\hline
\end{tabular}

Table 5. Post first line chemotherapy course in PMNSGCT.

\begin{tabular}{|l|c|}
\hline & Nonseminoma $(\boldsymbol{n}=\mathbf{3 4})$ \\
\hline Surveillance & $6(17.6 \%)$ \\
\hline Died before reassessment & $2(5.8 \%)$ \\
\hline Surgical resection & $12(32.4 \%)$ \\
\hline - R0 & 4 \\
\hline - R1 & 2 \\
\hline - R2 & 3 \\
\hline - No informationa & 3 \\
\hline Surgical histopathology $(n=12)$ & \\
- No residual tumour & $5(41.6 \%)$ \\
- Residual tumour & $2(16.6 \%)$ \\
- Teratoma & $2(16.6 \%)$ \\
\hline Salvage chemotherapy & $3(25.2 \%)$ \\
\hline
\end{tabular}

asurgery done at another centre

\section{Salvage therapy}

Sixteen patients with PMNSGCT received salvage chemo for refractory disease or disease progression/relapse. Two patients received TIP chemotherapy, one received Gem-ox chemotherapy and 11 patients received Vinblastin, ifosfamide and cisplatin chemotherapy. The median number of cycles was 3 . Five out of these 16 patients underwent surgery after salvage chemotherapy. There was viable tumour on histopathology in one patient, no tumour in three patients and one patient had surgery elsewhere. None underwent HDT and ASCT.

\section{Survival outcomes}

The median follow up for the entire patient cohort was 10.54 months (range: 1-178 months).

Seminoma: There was no EFS events in this cohort. The median EFS was not reached (range: 1-177 months) and all patients were alive at 2 years.

Nonseminomatous tumours: There were 19 (51.4\%) progression-free survival (PFS) events and 16 deaths (43.2\%) among the entire cohort of 37 patients. The median OS was 9.06 months and median PFS was 4.63 months. The proportion of patients alive at 1 year and 2 years were $35 \%$ and $24.3 \%$, respectively. The details are shown in Figures 2 and 3. 


\section{Discussion}

Primary GCTs of the mediastinum are associated with a poor prognosis when compared to gonadal GCTs [2, 5]. Even in developed nations, the multidisciplinary management of these tumours poses a challenge. Though GCTs among the adolescent and young population are treated with a curative intent worldwide, the additional challenges faced in LMIC settings are unique and include delayed presentation, higher proportion of treatment abandonment, poor follow up and limited access to more advanced salvage treatments like HDT and ASCT (HDT/SCT) $[6-8,11]$. Our study reports real world outcomes among patients with PMGCTs in a LMIC setting.

Mediastinal seminoma is associated with a good prognosis with infrequent relapses [12]. Our patients with mediastinal seminoma ( $n=20$ ) had a larger median tumour size at diagnosis and higher proportion of patients with 'intermediate risk' disease as per the IGCCCG stratification. All our patients with mediastinal seminoma were treated with BEP/EP chemotherapy and consistent with prior literature, had the best outcomes with a median EFS of 24.39 months with no relapses during the follow up period. The incidence of false positivity on EOT PET CT was high and active surveillance is an acceptable option for these patients [13].

Our patients with PMNSGCT $(n=37)$ had more advanced disease at diagnosis as reflected by higher mean AFP/HCG, higher incidence of extra mediastinal disease and distant metastases. All were stratified as 'poor risk' based on the IGCCCG risk stratification. These factors are well recognised as predictors of poor survival outcomes and disease recurrence [14]. We used BEP induction chemotherapy for four cycles for majority of our patients and saw mostly partial responses. We noted that even though tumour markers plateaued at the end of first line chemotherapy with minimal radiological regression, these tumours became more cystic and necrotic.

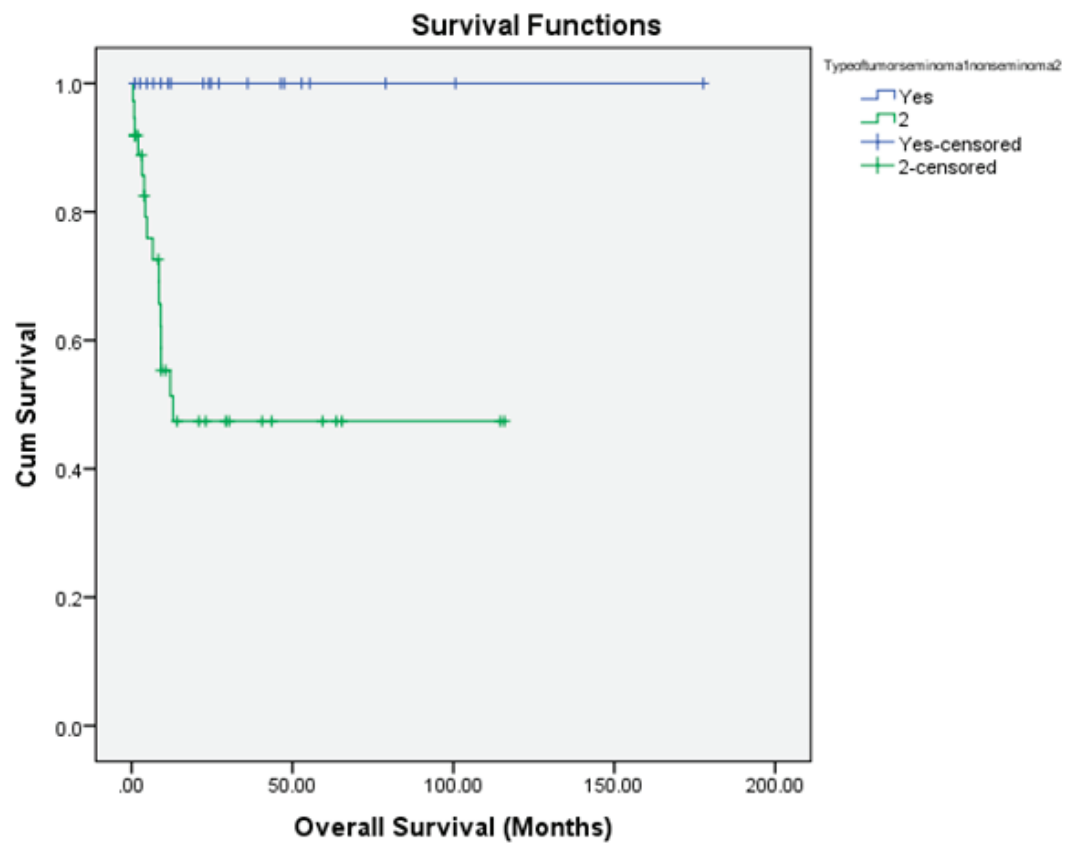

Figure 2. Kaplan-Meier OS estimate according to histology (blue line: seminoma and green line: PMNSGCT). 
(a)

Seminoma

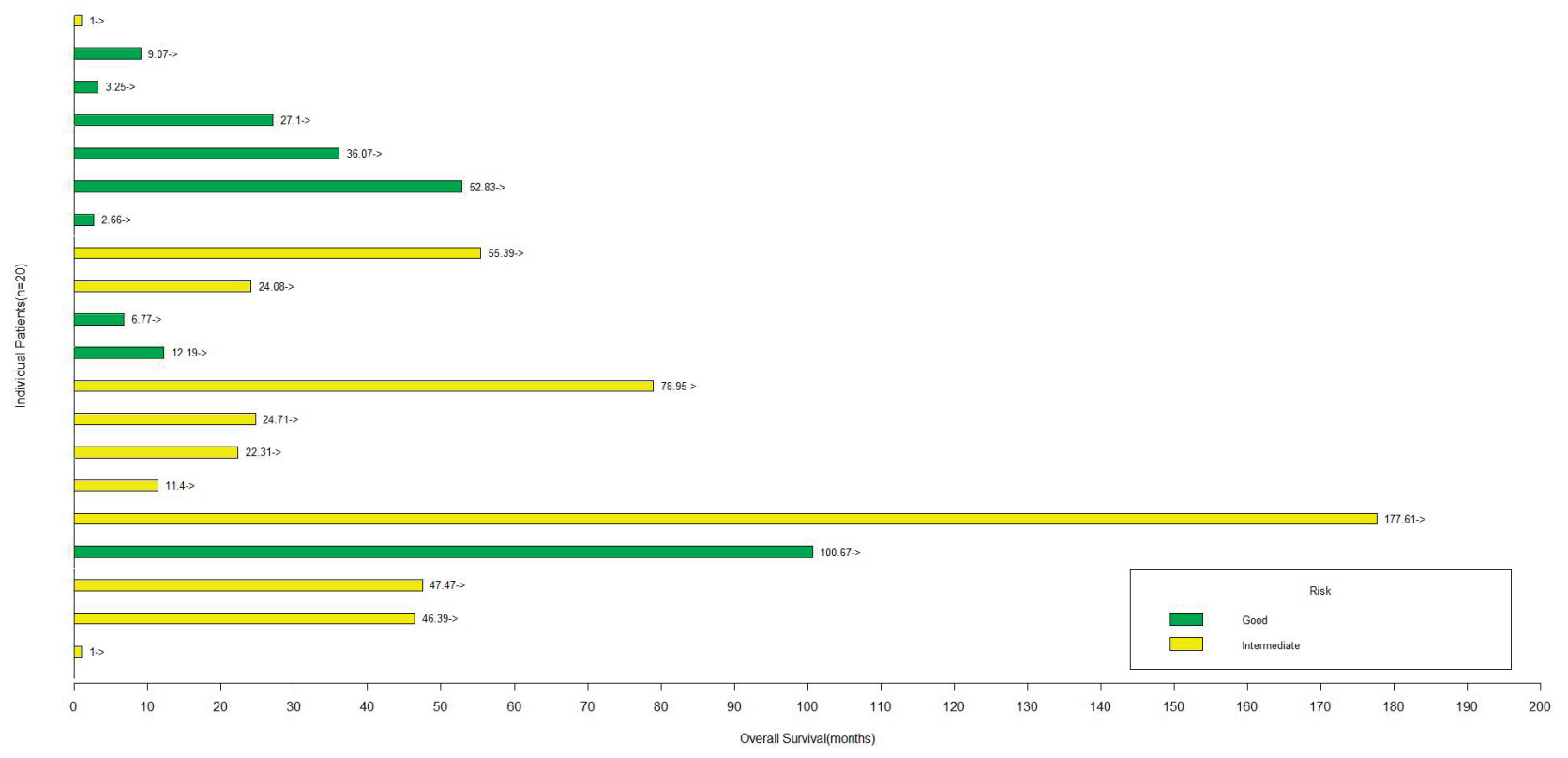

(b)

PMNSGCT

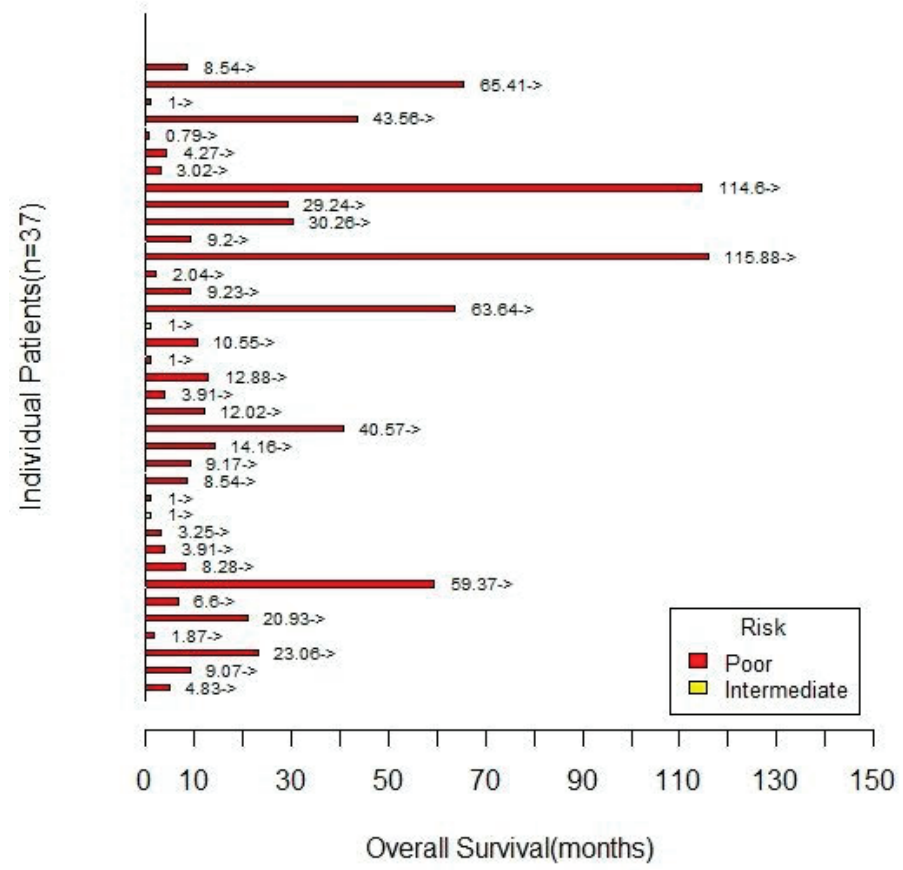

Figure 3. (a): Swimmer's plot depicting OS for patients with mediastinal seminoma. (b): Swimmer's plot depicting OS for patients with PMNSGCT. 
The role of surgery following chemotherapy is crucial to direct subsequent therapeutic decisions [15]. In addition to quantifying response to induction chemo, surgery also helps debulk chemo resistant tumour; especially when this is limited to the mediastinum. Single centre experiences reported from Indiana University and Memorial Sloan Kettering Cancer Center best illustrate the role of post chemotherapy surgery towards improving survival outcomes and cure rates in PMNSGCT including a subset of chemo refractory patients with localised intra-thoracic disease, if their mediastinal primary is resectable [15-18]. Approximately a third of our patients with PMNSGCT underwent surgical resection. There was residual viable tumour in $16.6 \%$ of our patients; in contrast to the higher rates described (30\%-54.8\%) [15-20].

We observed that the incidence of haematological toxicity (i.e.) anaemia (all grades and G3/4) and thrombocytopenia (all grades and G3/4) were high. The rates of febrile neutropenia were high in both seminoma and PMNSGCT in comparison to Western data. Compared to poor risk patients with gonadal primary GCTs and lung metastases, the need and extent of pulmonary surgery among patients with extra gonadal NSGCTs is much more extensive and may hence predispose these patients to a greater risk of drug induced pneumonitis [15]. There is a debate on whether omitting bleomycin among these patients who are planned for a major thoracic surgery consisting of thoracotomy followed by surgical excision of these tumours, reduces perioperative pulmonary complications [15, 21]. There was no severe bleomycin related pulmonary toxicity among our patients; this could be an underestimation since DLCOs (diffusing capacity for carbon monoxide) were not routinely monitored before and after chemotherapy.

The survival outcomes among patients with PMNSGCT worldwide have not changed dramatically even in the post cisplatin era [9, 14-18, 22, 23]. Despite improvements in early diagnosis, availability of cisplatin-based chemotherapy, advancements in surgical expertise and SCT, the 5 year OS has not crossed the $60 \%$ mark [14-18, 22, 23]. It is also evident that patients undergoing surgical resection have a better outcome when compared to those who did not undergo surgery $[17,18]$.

In the Asian subcontinent, Liu et al [22] have also shown that though the 5-year survival among patients with primary mediastinal seminoma is $87 \%$, the 5 year survival among patients with nonseminomatous mediastinal GCTs was only $27 \%$. However, our outcomes among PMNSGCT in the real world LMIC setting are worse; with median OS 9.06 months and median PFS 4.63 months. The proportion of our patients alive at 1 year and 2 years is also inferior to those described a priori [9].

Even though 16 patients received salvage/second line chemotherapy, none received HDT/ASCT; which has been shown to be acceptable as a second line strategy and has produced long standing remissions [24-26]. The lack of targetable mutations and lack of efficacy of immunotherapy among patients with relapsed/refractory GCTs coupled with the higher proportion of patients presenting with adverse prognostic factors at diagnosis including extra thoracic disease, indicate the importance of an intensive first line treatment approach inclusive of surgical resection, if a curative intent of treatment is to be attempted [4, 5]. Also, patients who required salvage chemotherapy prior to surgery have an inferior PFS when compared to those who did not require salvage chemotherapy prior to surgery $[17,18]$.

In LMIC settings like ours, the management of GCTs is more challenging, given the higher rates of treatment abandonment as seen among our patients with testicular cancer in India; despite their curative intent of treatment [6]. This is attributed to the predominance of younger patients, some of whom have to support a dependent family and the need to often travel long distances for their treatment. We acknowledge that our study is limited by its retrospective nature, small sample size, short follow up durations and limited interpretation of toxicity data. However, for rare tumours like PMGCTs, generation of real-world data plays a significant role in further refining our therapeutic approach toward the multimodality management of these patients and also highlights the need for collaboration among high volume centres; especially in LMIC settings; in order to improve our outcomes.

\section{Conclusion}

In our study, patients with PMNSGCT had a poorer outcome in comparison to mediastinal seminoma. This was predominantly due to a higher proportion of patients presenting with extra-thoracic disease and inability to achieve a complete surgical resection among patients with extra mediastinal disease. Outcomes in mediastinal seminoma are favourable; with chemotherapy alone producing excellent cure rates. 


\section{Conflicts of interest}

All authors have no conflicts of interest to declare.

\section{Funding}

The authors have not received any financing to carry out this work.

\section{Authors' contributions}

Anjana Joel: conceptualization, methodology, formal analysis, investigation, writing - original draft, visualization.

Namrata Mathew: conceptualization, methodology, formal analysis, investigation, writing - review \& editing, visualization, supervision, project administration, funding acquisition.

Shalom Sylvester Andugala: conceptualization, methodology, formal analysis, writing - review \& editing, visualization.

Sherin Daniel: conceptualization, methodology, formal analysis, investigation, writing - review \& editing, visualization, supervision.

Birla Roy Gnanamuthu: conceptualization, methodology, formal analysis, investigation, writing - review \& editing, visualization.

Ajoy Oommen John: conceptualization, methodology, investigation, writing - review \& editing, visualization.

Josh Thomas Georgy: conceptualization, methodology, investigation, writing - review \& editing, visualization.

Raju Titus Chacko: methodology, formal analysis, investigation, writing - review \& editing, supervision, visualization.

Aparna Irodi: methodology, formal analysis, investigation, writing - review \& editing, visualization.

Bijesh Yadav: formal analysis, data curation, software.

Subhashini John: methodology, formal analysis, investigation, writing - review \& editing, visualization.

Ashish Singh: writing - review \& editing, supervision, project administration, funding acquisition, resources.

\section{Acknowledgments}

The authors' would like to thank Mr Anurag Joel for technical assistance.

\section{References}

1. Hainsworth JD and Greco FA (1992) Extragonadal germ cell tumors and unrecognized germ cell tumors Semin Oncol 19(2) 119-127 PMID: 1313190

2. International Germ Cell Cancer Collaborative Group (2019) International Germ Cell Consensus Classification: a prognostic factorbased staging system for metastatic germ cell cancers J Clin Oncol [cited 2019 Dec 26] [https://ascopubs.org/doi/pdf/10.1200/ JCO.1997.15.2.594]

3. Necchi A, Bratslavsky G, and Chung J, et al (2019) Genomic features for therapeutic insights of chemotherapy-resistant, primary mediastinal nonseminomatous germ cell tumors and comparison with gonadal counterpart Oncologist 24(4) e142-e145 https://doi. org/10.1634/theoncologist.2018-0430 PMID: 30659078 PMCID: 6459248 
4. Adra N, Einhorn LH, and Althouse SK, et al (2018) Phase II trial of pembrolizumab in patients with platinum refractory germ-cell tumors: a Hoosier Cancer Research Network Study GU14-206 Ann Oncol 29(1) 209-214 https://doi.org/10.1093/annonc/mdx680

5. Rosti G, Secondino S, and Necchi A, et al (2019) Primary mediastinal germ cell tumors Semin Oncol 46(2) 107-111 https://doi. org/10.1053/j.seminoncol.2019.04.001 PMID: 31076171

6. Saju SV, Radhakrishnan V, and Ganesan TS, et al (2019) Factors that impact the outcomes in testicular germ cell tumors in low-middleincome countries Med Oncol 36(3) 2 https://doi.org/10.1007/s12032-019-1252-6

7. Mishra S, Das Majumdar SK, and Sable M, et al (2020) Primary malignant mediastinal germ cell tumors: a single institutional experience South Asian J Cancer 9(1) 27-29 https://doi.org/10.4103/sajc.sajc_47_19 PMID: 31956616 PMCID: 6956594

8. Joshi A, Zanwar S, and Shetty N, et al (2016) Epidemiology of male seminomatous and nonseminomatous germ cell tumors and response to first-line chemotherapy from a tertiary cancer center in India Indian J Cancer 53(2) 313. https://doi.org/10.4103/0019-509X.197741

9. Biswas B, Dabkara D, and Sengupta M, et al (2020) Multimodality treatment outcome in patients with primary malignant mediastinal germ cell tumor in adults Cancer Rep e1306

10. Mostert S, Arora RS, and Arreola M, et al (2011) Abandonment of treatment for childhood cancer: position statement of a SIOP PODC Working Group Lancet Oncol 12(8) 719-720 https://doi.org/10.1016/S1470-2045(11)70128-0 PMID: 21719348

11. Raina V, Shukla NK, and Rath GK, et al (1993) Clinical profile and problems of management of 108 cases of germ cell tumours of testis at Institute Rotary Cancer Hospital, All India Institute of Medical Sciences New Delhi 1985-1990 Br J Cancer 67(3) 573-577 https:// doi.org/10.1038/bjc.1993.105 PMID: 8439506 PMCID: 1968273

12. Hartmann JT, Nichols CR, and Droz J-P, et al (2002) Prognostic variables for response and outcome in patients with extragonadal germcell tumors Ann Oncol 13(7) 1017-1028 https://doi.org/10.1093/annonc/mdf176 PMID: 12176779

13. Cathomas R, Klingbiel D, and Bernard B, et al (2018) Questioning the value of fluorodeoxyglucose positron emission tomography for residual lesions after chemotherapy for metastatic seminoma: results of an international global germ cell cancer group registry $\mathrm{J}$ Clin Oncol 36(34) 3381-3387 https://doi.org/10.1200/JCO.18.00210

14. Rivera C, Arame A, and Jougon J, et al (2010) Prognostic factors in patients with primary mediastinal germ cell tumors, a surgical multicenter retrospective study Interact Cardiovasc Thorac Surg 11(5) 585-589 https://doi.org/10.1510/icvts.2010.238717 PMID: 20709694

15. Kesler KA, Rieger KM, and Hammoud ZT, et al (2008) A 25-year single institution experience with surgery for primary mediastinal nonseminomatous germ cell tumors Ann Thorac Surg 85(2) 371-378 https://doi.org/10.1016/j.athoracsur.2007.09.020 PMID: 18222228

16. Sarkaria IS, Bains MS, and Sood S, et al (2011) Resection of primary mediastinal non-seminomatous germ cell tumors: a 28-year experience at Memorial Sloan-Kettering Cancer Center J Thorac Oncol 6(7) 1236-1241 https://doi.org/10.1097/JTO.0b013e31821d513e PMID: 21610519

17. Kesler KA, Stram AR, and Timsina LR, et al (2020) Outcomes following surgery for primary mediastinal nonseminomatous germ cell tumors in the cisplatin era [Internet] J Thorac Cardiovasc Surg [https://pubmed.ncbi.nlm.nih.gov/32446546/] Date accessed: 14/10/20 https://doi.org/10.1016/j.jtcvs.2020.01.118

18. Caso R, Jones G, and Bains M, et al (2020) Outcomes after multidisciplinary management of primary mediastinal germ cell tumors Ann Surg [Internet] [insights.ovid.com] Date accessed: 14/10/2014/10/20 https://doi.org/10.1097/SLA.0000000000003754

19. Vuky J, Bains M, and Bacik J, et al (2001) Role of postchemotherapy adjunctive surgery in the management of patients with nonseminoma arising from the mediastinum J Clin Oncol 19 682-688 https://doi.org/10.1200/JCO.2001.19.3.682 PMID: 11157018

20. Kesler KA, Rieger KM, and Ganjoo KN, et al (1999) Primary mediastinal nonseminomatous germ cell tumors: the influence of postchemotherapy pathology on longterm survival after surgery J Thorac Cardiovasc Surg 118 692-670 https://doi.org/10.1016/SO0225223(99)70015-2 PMID: 10504636 
21. Ranganath P, Kesler KA, and Einhorn LH (2016) Perioperative morbidity and mortality associated with bleomycin in primary mediastinal nonseminomatous germ cell tumor J Clin Oncol 34(36) 4445-4446 https://doi.org/10.1200/JC0.2016.69.8910 PMID: 27621392

22. Liu Y, Wang Z, and Peng Z-M, et al (2014) Management of the primary malignant mediastinal germ cell tumors: experience with 54 patients Diagn Pathol 9(1) 33 https://doi.org/10.1186/1746-1596-9-33 PMID: 24552239 PMCID: 3996080

23. Wang L, Zhao J, and An T, et al (2020) Clinical characteristics and outcomes of patients with primary mediastinal germ cell tumors: a single-center experience Front Oncol [Internet] 101137 [https://www.frontiersin.org/articles/10.3389/fonc.2020.01137/full] Date accessed: 24/08/20 https://doi.org/10.3389/fonc.2020.01137

24. De Giorgi U, Demirer T, and Wandt H, et al (2005) Second-line high-dose chemotherapy in patients with mediastinal and retroperitoneal primary non-seminomatous germ cell tumors: the EBMT experience Ann Oncol Off J Eur Soc Med Oncol 16(1) 146-151 https://doi. org/10.1093/annonc/mdi017

25. Bokemeyer C, Schleucher N, and Metzner B, et al (2003) First-line sequential high-dose VIP chemotherapy with autologous transplantation for patients with primary mediastinal nonseminomatous germ cell tumours: a prospective trial Br J Cancer 89(1) 29-35 https:// doi.org/10.1038/sj.bjc.6600999 PMID: 12838296 PMCID: 2394224

26. Einhorn LH and Brames MJ (2007) High-dose chemotherapy and stem-cell rescue for metastatic germ-cell tumors N Engl J Med 9 